Comment. Math. Helv. 73 (1998) 566-583

0010-2571/98/040566-18 \$1.50+0.20/0
(C) 1998 Birkhäuser Verlag, Basel

Commentarii Mathematici Helvetici

\title{
Fano contact manifolds and nilpotent orbits
}

\author{
Arnaud Beauville*
}

\begin{abstract}
A contact structure on a complex manifold $M$ is a corank 1 subbundle $F$ of $T_{M}$ such that the bilinear form on $F$ with values in the quotient line bundle $L=T_{M} / F$ deduced from the Lie bracket of vector fields is everywhere non-degenerate. In this paper we consider the case where $\mathrm{M}$ is a Fano manifold; this implies that $\mathrm{L}$ is ample.

If $\mathfrak{g}$ is a simple Lie algebra, the unique closed orbit in $\mathbf{P}(\mathfrak{g})$ (for the adjoint action) is a Fano contact manifold; it is conjectured that every Fano contact manifold is obtained in this way. A positive answer would imply an analogous result for compact quaternion-Kahler manifolds with positive scalar curvature, a longstanding question in Riemannian geometry.

In this paper we solve the conjecture under the additional assumptions that the group of contact automorphisms of $\mathrm{M}$ is reductive, and that the image of the rational map $\mathrm{M} \rightarrow-\rightarrow$ $\mathbf{P}\left(H^{0}(\mathrm{M}, \mathrm{L})^{*}\right)$ associated to $\mathrm{L}$ has maximum dimension. The proof relies on the properties of the nilpotent orbits in a semi-simple Lie algebra, in particular on the work of R. Brylinski and B. Kostant.
\end{abstract}

Mathematics Subject Classification (1991). Primary 14J45, 53C15; Secondary 14L30, $14 \mathrm{M} 17$.

Keywords. Contact structure, Fano manifolds, quaternion-Kähler manifolds, nilpotent orbits, contact moment map.

\section{Introduction}

A contact structure on a complex manifold $\mathrm{M}$ is a corank 1 subbundle $\mathrm{F} \subset \mathrm{T}_{\mathrm{M}}$ such that the bilinear form on $F$ with values in the quotient line bundle $L=T_{M} / F$ deduced from the Lie bracket on $\mathrm{T}_{\mathrm{M}}$ is everywhere non-degenerate. This implies that the dimension of $\mathrm{M}$ is odd, say $\operatorname{dim} \mathrm{M}=2 n+1$, and that the canonical bundle $\mathrm{K}_{\mathrm{M}}$ is isomorphic to $\mathrm{L}^{-n-1}$. In this paper we will consider the case where $\mathrm{M}$ is compact and $\mathrm{L}$ is ample, that is, $\mathrm{M}$ is a Fano manifold.

This turns out to be a strong restriction on the manifold $\mathrm{M}$; the only examples known so far are obtained as follows (see Prop. 2.6 and 2.2 below). Let $\mathfrak{g}$ be a simple complex Lie algebra; the adjoint group acting on $\mathbf{P}(\mathfrak{g})$ has exactly one closed orbit $\mathbf{P} \mathcal{O}_{\min }$, which is the projectivization of the minimal nilpotent orbit

* Partially supported by the European HCM project "Algebraic Geometry in Europe" (AGE). 
$\mathcal{O}_{\text {min }} \subset \mathfrak{g}$. The Kostant-Kirillov symplectic structure on $\mathcal{O}_{\text {min }}$ defines a contact structure on $\mathbf{P} \mathcal{O}_{\min }$.

It is generally conjectured that every Fano contact manifold is obtained in this way. This problem is motivated by Riemannian geometry, more precisely by the study of compact quaternion-Kähler manifolds. I will say only a few words here, referring for instance to [L-S], [L] and the bibliography therein for a more complete treatment. A quaternion-Kähler manifold $\mathrm{Q}$ is a Riemannian manifold with holonomy $\quad(n) \quad(1)$. It carries a natural $\mathrm{S}^{2}$-bundle $\mathrm{M} \rightarrow \mathrm{Q}$, the twistor space, which turns out to be a complex contact manifold; moreover if $\mathrm{Q}$ is compact and its scalar curvature is positive, $\mathrm{M}$ is a Fano contact manifold. The only known examples of positive quaternion-Kähler manifolds are certain symmetric spaces associated to each compact simple Lie group, the so-called "Wolf spaces"; thanks to the work of LeBrun and Salamon, a positive answer to the above conjecture would imply that every compact quaternion-Kähler manifold with positive scalar curvature is isometric to a Wolf space.

Our result is the following:

Theorem 0.1. Let $\mathrm{M}$ be a Fano contact manifold, satisfying the following conditions:

(H1) The rational map $\varphi_{\mathrm{L}}: \mathrm{M} \rightarrow \mathbf{P}\left(\mathrm{H}^{0}(\mathrm{M}, \mathrm{L})^{*}\right)$ associated to the line bundle $\mathrm{L}$ is generically finite (that is, $\left.\operatorname{dim} \varphi_{\mathrm{L}}(\mathrm{M})=\operatorname{dim} \mathrm{M}\right)$;

(H2) The group $\mathrm{G}$ of contact automorphisms of $\mathrm{M}$ is reductive.

Then the Lie algebra $\mathfrak{g}$ of $\mathrm{G}$ is simple, and $\mathrm{M}$ is isomorphic to the minimal orbit $\mathbf{P} \mathcal{O}_{\min } \subset \mathbf{P}(\mathfrak{g})$.

While hypothesis (H1) is rather strong, (H2) is harmless from the point of view of Riemannian geometry: by the results of [L], it always holds for the twistor spaces of positive quaternion-Kähler manifolds.

We will get an apparently stronger result, namely that $\mathrm{M}$ and $\mathbf{P} \mathcal{O}_{\text {min }}$ are isomorphic as contact complex manifolds. It is however a general fact that whenever two compact simply-connected contact manifolds are isomorphic, the isomorphism can be chosen compatible with the contact structures ([L], Prop. 2.3).

The strategy of the proof is as follows. Using some elementary symplectic geometry, the map $\varphi_{\mathrm{L}}$ can be viewed as a "contact moment map" $\mathrm{M} \rightarrow \mathbf{P}(\mathfrak{g})$. Then (H1) implies that $\mathrm{G}$ has an open orbit in $\mathrm{M}$, whose image by $\varphi_{\mathrm{L}}$ is a nilpotent orbit $\mathbf{P O} \subset \mathbf{P}(\mathfrak{g})$. We are thus led to classify finite G-equivariant coverings $\mathrm{M} \rightarrow \overline{\mathbf{P O}}$, where $\mathrm{M}$ is smooth. Examples of such coverings appear in $[\mathrm{B}-\mathrm{K}]$, with $\mathrm{M}$ being the minimal orbit in $\mathbf{P}\left(\mathfrak{g}^{\prime}\right)$ for some simple Lie algebra $\mathfrak{g}^{\prime}$ containing $\mathfrak{g}$; our key result is that all possible examples arise essentially in this way. Theorem 0.1 follows then easily. 


\section{Contact geometry}

Let $\mathrm{M}$ be a complex contact projective manifold. Recall that the contact structure is given by an exact sequence

$$
0 \rightarrow \mathrm{F} \longrightarrow \mathrm{T}_{\mathrm{M}} \stackrel{\theta}{\longrightarrow} \mathrm{L} \rightarrow 0
$$

such that the $\left(\mathcal{O}_{\mathrm{M}}\right.$-bilinear $)$ alternate form $(X, Y) \mapsto \theta([X, Y])$ on $\mathrm{F}$ is everywhere non-degenerate. Alternatively the contact structure can be described by the twisted 1-form $\theta \in \mathrm{H}^{0}\left(\mathrm{M}, \Omega_{\mathrm{M}}^{1} \otimes \mathrm{L}\right)$, the contact form.

We denote by $\mathrm{G}$ the neutral component of the group of automorphisms of $\mathrm{M}$ preserving $\mathrm{F}$. This is an algebraic group, whose Lie algebra $\mathfrak{g}$ consists of the vector fields $X \in \mathrm{H}^{0}\left(\mathrm{M}, \mathrm{T}_{\mathrm{M}}\right)$ such that $[X, \mathrm{~F}] \subset \mathrm{F}$. The following result is well-known (see e.g. [L]):

Proposition 1.1. The map $\mathrm{H}^{0}(\theta): \mathrm{H}^{0}\left(\mathrm{M}, \mathrm{T}_{\mathrm{M}}\right) \rightarrow \mathrm{H}^{0}(\mathrm{M}, \mathrm{L})$ maps $\mathfrak{g}$ isomorphically onto $\mathrm{H}^{0}(\mathrm{M}, \mathrm{L})$.

Proof. Let us first prove the decomposition $\mathrm{H}^{0}\left(\mathrm{M}, \mathrm{T}_{\mathrm{M}}\right)=\mathrm{H}^{0}(\mathrm{M}, \mathrm{F}) \oplus \mathfrak{g}$. Let $X \in \mathrm{H}^{0}\left(\mathrm{M}, \mathrm{T}_{\mathrm{M}}\right)$. The map $U \mapsto \theta([X, U])$ from $\mathrm{F}$ to $\mathrm{L}$ is $\mathcal{O}_{\mathrm{M}}$-linear, hence there exists a unique vector field $X^{\prime}$ in $\mathrm{F}$ such that $\theta([X, U])=\theta\left(\left[X^{\prime}, U\right]\right)$ for all $U$ in $\mathrm{F}$. This means that $\left[X-X^{\prime}, U\right]$ belongs to $\mathrm{F}$, that is that $X-X^{\prime}$ belongs to $\mathfrak{g}$. Writing $X=X^{\prime}+\left(X-X^{\prime}\right)$ provides the required direct sum decomposition.

Let $\mathcal{L} \subset \mathrm{T}_{\mathrm{M}}$ be the subsheaf of infinitesimal contact transformations. Applying the above result to each open subset of $\mathrm{M}$ we get $\mathrm{T}_{\mathrm{M}}=\mathrm{F} \oplus \mathcal{L}$, so that $\theta$ induces a ( $\mathbf{C}$-linear) isomorphism of $\mathcal{L}$ onto $\mathrm{L}$. Our statement follows by taking global sections.

(1.2) For each $g \in \mathrm{G}$ the automorphism $\mathrm{T}(g)$ of $\mathrm{T}_{\mathrm{M}}$ induces an automorphism of $\mathrm{L}$ above $g$; in other words, the line bundle $\mathrm{L}$ has a canonical Glinearization. In particular the group $\mathrm{G}$ acts on $\mathrm{H}^{0}(\mathrm{M}, \mathrm{L})$; the isomorphism $\theta: \mathfrak{g} \rightarrow \mathrm{H}^{0}(\mathrm{M}, \mathrm{L})$ is $\mathrm{G}$-equivariant with respect to this action and the adjoint action on $\mathfrak{g}$. Also the rational map $\varphi_{\mathrm{L}}: \mathrm{M} \rightarrow \mathbf{P}\left(\mathrm{H}^{0}(\mathrm{M}, \mathrm{L})^{*}\right)$ associated to the line bundle $\mathrm{L}$ is $\mathrm{G}$-equivariant.

(1.3) Let $\mathrm{L}^{\times}$be the principal $\mathbf{C}^{*}$-bundle associated to the dual line bundle $\mathrm{L}^{*}$ - that is the complement of the zero section in $\mathrm{L}^{*}$, on which $\mathbf{C}^{*}$ acts by homotheties. We will say that a $p$-form $\omega$ on $L^{\times}$is $\mathbf{C}^{*}$-equivariant if $\lambda^{*} \omega=\lambda \omega$ for every $\lambda \in \mathbf{C}^{*}$.

Let us denote by $p$ the projection of $\mathrm{L}^{*}$ onto $\mathrm{M}$. We have a canonical linear form $\tau: p^{*} \mathrm{~L} \rightarrow \mathcal{O}_{\mathrm{L}^{*}}$, which is bijective on $\mathrm{L}^{\times}$: if $s$ is a local section of $\mathrm{L}$ on $\mathrm{M}$, the function $\tau\left(p^{*} s\right)$ maps a point $(m, \xi)$ of $\mathrm{L}^{*}\left(\xi \in \mathrm{L}(m)^{*}\right)$ to $\langle s(m), \xi\rangle$. We use $\tau$ to trivialize $p^{*} \mathrm{~L}$ on $\mathrm{L}^{\times}$. We can therefore consider $p^{*} \theta$ as a 1 -form on $\mathrm{L}^{\times}$; it is $\mathbf{C}^{*}$-equivariant. The following lemma is classical (see for instance [A], App. 4 E, or [L], p. 425): 
Lemma 1.4. The 2 -form $d\left(p^{*} \theta\right)$ is a symplectic structure on $\mathrm{L}^{\times}$. Conversely, any $\mathbf{C}^{*}$-equivariant symplectic 2 -form on $\mathrm{L}^{\times}$is of the form $d\left(p^{*} \theta\right)$, where $\theta$ is a contact form on $\mathrm{M}$, which is uniquely determined.

(1.5) To each point $(m, \xi)$ of $\mathrm{L}^{*}\left(m \in \mathrm{M}, \xi \in \mathrm{L}(m)^{*}\right)$, we associate the linear form $\mu_{\mathrm{L}}(m, \xi)$ on $\mathrm{H}^{0}(\mathrm{M}, \mathrm{L})$ defined by $\left\langle\mu_{\mathrm{L}}(m, \xi), s\right\rangle=\langle s(m), \xi\rangle$ for each $s \in \mathrm{H}^{0}(\mathrm{M}, \mathrm{L})$. This gives a morphism $\mu_{\mathrm{L}}: \mathrm{L}^{*} \rightarrow \mathrm{H}^{0}(\mathrm{M}, \mathrm{L})^{*}$ which is $\mathbf{C}^{*}$-equivariant and induces on the projectivizations the rational map $\varphi_{\mathrm{L}}: \mathrm{M}-\rightarrow \mathbf{P}\left(\mathrm{H}^{0}(\mathrm{M}, \mathrm{L})^{*}\right)$. Using the isomorphism $\theta: \mathfrak{g} \stackrel{\sim}{\longrightarrow} \mathrm{H}^{0}(\mathrm{M}, \mathrm{L})$ (Prop. 1.1), we get a commutative Gequivariant diagram

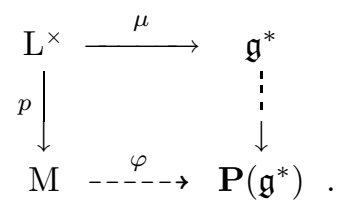

As we have seen in (1.2), the action of $\mathrm{G}$ on $\mathrm{M}$ lifts to an action on $\mathrm{L}^{\times}$, which is linear on the fibres; as a consequence, any field $X \in \mathfrak{g}$ lifts to a vector field $\widetilde{X}$ on $\mathrm{L}^{\times}$which projects to $X$ on $\mathrm{M}$.

Proposition 1.6. $\mu$ is a moment map for the action of $\mathrm{G}$ on the symplectic manifold $\mathrm{L}^{\times}$.

Proof. This means by definition that for each $X \in \mathfrak{g}$, the vector field $\widetilde{X}$ is the Hamiltonian vector field associated to the function $\langle\mu, X\rangle$ on $\mathrm{L}^{\times}$. To prove this, we first observe that since the 1 -form $\eta=p^{*} \theta$ is preserved by $\mathrm{G}$, its Lie derivative $\mathrm{L}_{\widetilde{X}} \eta$ vanishes for each $X \in \mathfrak{g}$. By the Cartan homotopy formula, this implies $i(\widetilde{X}) d \eta=-d\langle\eta, \widetilde{X}\rangle$. But we have $\langle\eta, \widetilde{X}\rangle=\tau\left(p^{*} \theta(X)\right)=\langle\mu, X\rangle$, thus $i(\widetilde{X}) d \eta=-d\langle\mu, X\rangle$, which proves our claim.

The classical computation of the differential of the moment map gives:

Proposition 1.7. Let $m \in \mathrm{M}$, and $\xi$ a point of $\mathrm{L}^{\times}$above $m$. The following conditions are equivalent:

(i) $\varphi$ is defined at $m$ and its differential $\mathrm{T}_{m}(\varphi)$ is injective;

(ii) the $\mathrm{G}$-orbit of $\xi$ is open in $\mathrm{L}^{\times}$;

(iii) the $\mathrm{G}$-orbit of $m$ is open in $\mathrm{M}$ and $\xi$ is conjugate under $\mathrm{G}$ to $\ell \xi$ for every $\ell \in \mathbf{C}^{*}$.

Proof. Since $\mu$ is $\mathbf{C}^{*}$-equivariant, condition (i) is equivalent to:

(i') $\mu(\xi) \neq 0$ and $\mathrm{T}_{\xi}(\mu)$ is injective.

Let $\omega$ be the symplectic 2 -form on $\mathrm{L}^{\times}$; for $v \in \mathrm{T}_{\xi}\left(\mathrm{L}^{\times}\right)$and $X \in \mathfrak{g}$, the 
formula $i(\widetilde{X}) \omega=-d\langle\mu, X\rangle$ (1.6) gives

$$
\left\langle\mathrm{T}_{\xi}(\mu) \cdot v, X\right\rangle=-\left\langle i(\widetilde{X}) \omega_{\xi}, v\right\rangle=\omega_{\xi}(v, \widetilde{X}(\xi)),
$$

so that the kernel of $\mathrm{T}_{\xi}(\mu)$ is the orthogonal of $\mathrm{T}_{\xi}(\mathrm{G} \cdot \xi)$ in $\mathrm{T}_{\xi}\left(\mathrm{L}^{\times}\right)$(with respect to $\omega_{\xi}$ ). This gives the equivalence of (i') and (ii); since the action of $\mathrm{G}$ commutes with the homotheties, (ii) is equivalent to (iii).

Corollary 1.8. a) If $\mathrm{L}$ is very ample, $\mathrm{M}$ is homogeneous.

b) If $\varphi$ is generically finite, $\mathrm{M}$ contains an open $\mathrm{G}$-orbit.

Proof. Under the hypothesis of a), each point of $\mathrm{M}$ has an open orbit, thus necessarily equal to $\mathrm{M}$. The hypothesis of $\mathrm{b}$ ) implies that $\varphi$ is an immersion at a general point of $\mathrm{M}$.

Cor. 1.8 a) has also been obtained by J. Wisniewski (private communication).

\section{Coadjoint orbits}

(2.1) Let $\mathfrak{g}$ be a Lie algebra; the adjoint group $\mathrm{G}$ acts on the dual $\mathfrak{g}^{*}$ of $\mathfrak{g}$ through the coadjoint representation. Recall that each coadjoint orbit $\mathcal{O}$ carries a canonical G-invariant symplectic structure $\Omega$, the Kostant-Kirillov structure: for $\xi \in \mathcal{O}$, the tangent space $\mathrm{T}_{\xi}(\mathcal{O})$ is canonically isomorphic to $\mathfrak{g} / \mathfrak{z}_{\xi}$, where $\mathfrak{z}_{\xi}=\operatorname{Ker}(\xi \circ \mathrm{ad})$ is the annihilator of $\xi$ in $\mathfrak{g}$; the 2 -form $\Omega_{\xi}$ is induced by the alternate form $(X, Y) \mapsto \xi([X, Y])$ on $\mathfrak{g}$. The following result shows that whenever $\mathcal{O}$ is invariant under homotheties, its image $\mathbf{P} \mathcal{O}$ in $\mathbf{P}\left(\mathfrak{g}^{*}\right)$ carries a natural contact structure:

Proposition 2.2. Let $\mathfrak{g}$ be a Lie algebra, $\mathrm{G}$ its adjoint group, $\xi$ a nonzero linear form on $\mathfrak{g}, \mathcal{O}$ its coadjoint orbit in $\mathfrak{g}^{*}, \mathbf{P} \mathcal{O}$ the image of $\mathcal{O}$ in $\mathbf{P}\left(\mathfrak{g}^{*}\right)$. The following conditions are equivalent:

(i) $\mathbf{P O}$ is odd-dimensional;

(ii) the orbit $\mathcal{O} \subset \mathfrak{g}^{*}$ is invariant by homotheties;

(iii) for each $\ell \in \mathbf{C}^{*}, \ell \xi$ is $\mathrm{G}$-conjugate to $\xi$;

(iv) there exists $H \in \mathfrak{g}$ such that $\xi \circ \operatorname{ad}(H)=\xi$;

(v) the annihilator $\mathfrak{z}_{\xi}$ of $\xi$ in $\mathfrak{g}$ is contained in $\operatorname{Ker} \xi$.

When these conditions are satisfied, the Kostant-Kirillov symplectic structure on $\mathcal{O}$ comes from a $\mathrm{G}$-invariant contact structure on $\mathbf{P} \mathcal{O}$.

(i) $\Leftrightarrow$ (iii): Let $\mathrm{Z}_{\xi}$ be the stabilizer of $\xi$ in $\mathrm{G}$, and $\mathrm{Z}_{[\xi]}$ the stabilizer of the image $[\xi]$ of $\xi$ in $\mathbf{P}\left(\mathfrak{g}^{*}\right)$. The action of $\mathrm{Z}_{[\xi]}$ on the line $[\xi]$ defines a homomorphism $\ell: \mathrm{Z}_{[\xi]} \rightarrow \mathbf{C}^{*}$, and we have an exact sequence

$$
0 \rightarrow \mathrm{Z}_{\xi} \longrightarrow \mathrm{Z}_{[\xi]} \stackrel{\ell}{\longrightarrow} \mathbf{C}^{*} \text {. }
$$


Since the orbit $\mathcal{O}$ is even-dimensional, (i) is equivalent to $\operatorname{dim} \mathrm{Z}_{[\xi]}=\operatorname{dim} \mathrm{Z}_{\xi}+1$, that is to the surjectivity of $\ell$, which is nothing but condition (iii).

(ii) $\Leftrightarrow$ (iii): Clear.

(iii) $\Leftrightarrow$ (iv): The Lie algebra $\mathfrak{z}_{[\xi]}$ of $\mathrm{Z}_{[\xi]}$ consists of the elements $H$ of $\mathfrak{g}$ such that $\xi \circ \operatorname{ad}(H)=\lambda \xi$ for some $\lambda=\lambda(H) \in \mathbf{C}$. The homomorphism $\lambda: \mathfrak{z}_{[\xi]} \rightarrow \mathbf{C}$ thus defined is the Lie derivative of $\ell$, so the surjectivity of $\ell$ is equivalent to the surjectivity of $\lambda$, that is to (iv).

(iv) $\Leftrightarrow(v)$ : The linear map $u: H \mapsto \xi \circ \operatorname{ad}(H)$ of $\mathfrak{g}$ into $\mathfrak{g}^{*}$ is antisymmetric, hence $\operatorname{Im} u=(\operatorname{Ker} u)^{\perp}$. But (iv) is equivalent to $\xi \in \operatorname{Im} u$ and (v) to $\xi \in(\operatorname{Ker} u)^{\perp}$.

Finally when $\mathcal{O}$ is invariant by homotheties, the Kostant-Kirillov 2 -form on $\mathcal{O}$ is $\mathbf{C}^{*}$-equivariant, and therefore comes from a G-invariant contact structure on $\mathbf{P} \mathcal{O}$ (lemma 1.4).

Remark 2.3. Assume that the equivalent conditions of Prop. 2.2 hold; the contact structure on $\mathbf{P O}$ can be described explicitely as follows. Let $\psi \in \mathcal{O}$; the tangent space $\mathrm{T}_{[\psi]}(\mathbf{P} \mathcal{O})$ is canonically isomorphic to $\mathfrak{g} / \mathfrak{z}[\psi]$. Observe that $\mathfrak{z}[\psi]$ is contained in $\operatorname{Ker} \psi$ : each element $\mathbf{Z}$ of $\mathfrak{z}[\psi]$ satisfies $\psi \circ \operatorname{ad}(\mathrm{Z})=\lambda \psi$ for some $\lambda \in \mathbf{C}$; if $\lambda=0$ we have $\psi(\mathrm{Z})=0$ by (v) above, while if $\lambda \neq 0$ we have $\psi(\mathrm{Z})=\lambda^{-1} \psi([\mathrm{Z}, \mathrm{Z}])=0$. Then the contact structure $\mathrm{F} \subset \mathrm{T}_{\mathbf{P O} \mathcal{O}}$ is defined by $\mathrm{F}_{[\psi]}=(\operatorname{Ker} \psi) / \mathfrak{z}[\psi] \cdot$

(2.4) Suppose that the Lie algebra $\mathfrak{g}$ is semi-simple. Using the Killing form we identify the G-module $\mathfrak{g}^{*}$ to $\mathfrak{g}$ endowed with the adjoint action. The element $\xi$ corresponds to a nonzero element $N$ of $\mathfrak{g}$. Conditions (iii) to (v) read:

(iii') for each $\ell \in \mathbf{C}^{*}, \ell N$ is G-conjugate to $N$;

(iv') there exists $H \in \mathfrak{g}$ such that $[H, N]=N$;

(v') the centralizer $\mathfrak{z}_{N}$ of $N$ in $\mathfrak{g}$ is orthogonal to $N$.

They are equivalent to $N$ being nilpotent: (iii') implies $\operatorname{Tr} \rho(N)^{p}=0$ for any representation $\rho$ of $\mathfrak{g}$ and any $p \geq 1$; conversely, if $N$ is nilpotent, (iv') holds by the Jacobson-Morozov theorem.

(2.5) Let $\mathfrak{h}$ be a Cartan subalgebra of $\mathfrak{g}, \mathrm{R}=\mathrm{R}(\mathfrak{g}, \mathfrak{h})$ the root system of $\mathfrak{g}$ relative to $\mathfrak{h}$. We have a direct sum decomposition $\mathfrak{g}=\mathfrak{h} \oplus \bigoplus_{\alpha \in \mathrm{R}} \mathfrak{g}^{\alpha}$. A nonzero vector $X_{\alpha} \in \mathfrak{g}^{\alpha}$ is called a root vector (relative to $\alpha$ ).

If $\mathfrak{g}$ is simple, the Weyl group acts transitively on the set of roots with a given length, and the corresponding root vectors are conjugate. This defines the (nilpotent) orbits $\mathcal{O}_{\min }$ of a long root vector and $\mathcal{O}_{\text {short }}$ of a short root vector; these orbits coincide if and only if all roots have the same length (types $\mathrm{A}_{l}, \mathrm{D}_{l}, \mathrm{E}_{l}$ ).

Proposition 2.6. Let $\mathfrak{g}$ be a simple complex Lie algebra. There exists exactly one closed orbit in $\mathbf{P}(\mathfrak{g})$ (for the adjoint action), namely the orbit $\mathbf{P} \mathcal{O}_{\min }$ of a long root vector. Every orbit contains $\mathbf{P} \mathcal{O}_{\min }$ in its closure. 
Proof. Let $N$ be a nonzero element of $\mathfrak{g}$. The orbit of $[N]$ in $\mathbf{P}(\mathfrak{g})$ is closed if and only if $\mathfrak{z}_{[N]}$ contains a Borel subalgebra $\mathfrak{b}$, so that there exists a linear form $\lambda$ on $\mathfrak{b}$ such that $\operatorname{ad}(X) \cdot N=\lambda(X) N$ for all $X \in \mathfrak{b}$. This means that $N$ is a highest weight vector for the adjoint representation; since $\mathfrak{g}$ is simple, the adjoint representation is irreducible, and its highest weight vector is $X_{\theta}$, where $\theta$ is the highest root with respect to the basis of $\mathrm{R}(\mathfrak{g}, \mathfrak{h})$ such that $\mathfrak{b}=\mathfrak{h} \oplus \oplus \mathfrak{g}^{\alpha}$. We

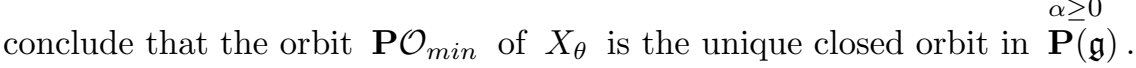

Examples 2.7. For the classical case, we get the following Fano contact manifolds:

$\mathrm{A}_{l}$ : the projectivized cotangent bundle $\mathbf{P} \mathrm{T}^{*}\left(\mathbf{P}^{l}\right)$;

$\mathrm{B}_{l}, \mathrm{D}_{l}$ : the Grassmannian $\mathbf{G}_{i s o}(2, \mathrm{~V})$ of isotropic 2-planes in a quadratic vector space $\mathrm{V}$, of dimension $2 l+1$ and $2 l$ respectively;

$\mathrm{C}_{l}$ : the projective space $\mathbf{P}^{2 l-1}$.

For the type $\mathrm{G}_{2}$ we get a Fano 5 -fold of index 3 which appears in the work of Mukai $[\mathrm{Mu}]$. The other exceptional Lie algebras give rise to Fano contact manifolds of dimension 15, 21, 33 and 57.

Remark 2.8. It follows from [L], Cor. 3.2, or from a direct computation, that if $\mathfrak{g}$ is not of type $\mathrm{C}_{l}$ the manifold $\mathbf{P} \mathcal{O}_{\min }$ admits a unique contact structure; in all cases, the contact structure we have defined is the unique G-invariant contact structure.

\section{First consequences of (H1) and (H2)}

(3.1) From now on we assume that $\varphi$ is generically finite (or equivalently, $\operatorname{dim} \varphi(\mathrm{M})=\operatorname{dim} \mathrm{M})$. By Cor. 1.8, this implies that $\mathrm{G}$ has an open orbit $\mathrm{M}^{\mathrm{O}}$ in $M$. Since $\varphi$ is G-equivariant, it is everywhere defined on $\mathrm{M}^{\mathrm{O}}$; the image $\varphi\left(\mathrm{M}^{\mathrm{O}}\right)$ is an orbit $\mathbf{P} \mathcal{O}$ of $\mathrm{G}$ in $\mathbf{P}\left(\mathfrak{g}^{*}\right)$, and the induced map $\varphi^{\mathrm{o}}: \mathrm{M}^{\mathrm{O}} \rightarrow \mathbf{P} \mathcal{O}$ is a finite étale covering.

Let us mention at once an immediate consequence: if a connected normal subgroup of $\mathrm{G}$ fixes a point $[\xi] \in \mathbf{P} \mathcal{O}$, it acts trivially on $\mathrm{M}^{\mathrm{o}}$, hence on $\mathrm{M}$; it follows that the stabilizer $\mathfrak{z}_{[\xi]}$ of $[\xi]$ in $\mathfrak{g}$ contains no nonzero ideal of $\mathfrak{g}$. In particular, the center of $\mathfrak{g}$ is trivial.

Lemma 3.2. Assume that the character group of $\mathrm{G}$ is trivial, and $\operatorname{Pic}(\mathrm{M})=\mathbf{Z}$. Then $\mathrm{M}-\mathrm{M}^{\mathrm{O}}$ has codimension $\geq 2$ in $\mathrm{M}$.

Proof. Let $m$ be a point of $\mathrm{M}^{\mathrm{o}},[\xi]$ its image in $\mathbf{P}\left(\mathfrak{g}^{*}\right)$. The stabilizer $\mathrm{Z}_{m}$ of $m$ in $\mathrm{G}$ is a subgroup of finite index in the stabilizer $\mathrm{Z}_{[\xi]}$ of $[\xi]$. Since $\mathrm{M}^{\mathrm{o}}$ and therefore $\mathbf{P O}$ are odd-dimensional, the equivalent conditions of Prop. 2.2 are 
satisfied; hence the homomorphism $\ell: \mathrm{Z}_{[\xi]} \rightarrow \mathbf{C}^{*}$ deduced from the action of $\mathrm{Z}_{[\xi]}$ on the line $[\xi]$ is surjective, and so is its restriction to $\mathrm{Z}_{m}$.

Recall that the group $\operatorname{Pic}^{G}\left(M^{\circ}\right)$ of $G$-linearized line bundles on $M^{\circ} \cong G / Z_{m}$ is canonically isomorphic to the character group $\mathbf{X}\left(\mathrm{Z}_{m}\right)$. On the other hand, the hypothesis on $G$ ensures that the forgetful map $\operatorname{Pic}^{G}\left(M^{o}\right) \rightarrow \operatorname{Pic}\left(M^{\circ}\right)$ is injective ([M], Ch. 1, Prop. 1.4). Since we have found a surjective character of $\mathrm{Z}_{m}$, it follows that $\operatorname{Pic}\left(\mathrm{M}^{\mathrm{O}}\right)$ contains an infinite cyclic group.

Let $\left(\mathrm{D}_{i}\right)_{i \in \mathrm{I}}$ be the family of one-codimensional components of $\mathrm{M}-\mathrm{M}^{\mathrm{O}}$. We have an exact sequence

$$
\mathbf{Z}^{\mathrm{I}} \stackrel{\left(\mathrm{D}_{i}\right)}{\longrightarrow} \operatorname{Pic}(\mathrm{M}) \longrightarrow \operatorname{Pic}\left(\mathrm{M}^{\mathrm{O}}\right) \rightarrow 0 \text {. }
$$

Since $\operatorname{Pic}(M)=\mathbf{Z}$ and each $\mathrm{D}_{i}$ has a nonzero class in $\operatorname{Pic}(\mathrm{M})$, the only possibility is $\mathrm{I}=\varnothing$.

Lemma 3.3. Let $\mathrm{M}$ be a normal projective variety, $\mathrm{L}$ an ample line bundle on $\mathrm{M}, \varphi: \mathrm{M} \rightarrow \mathbf{P}^{r}$ the associated rational map, $\mathrm{N} \subset \mathbf{P}^{r}$ its image. Assume that there are open subsets $\mathrm{M}^{\mathrm{O}} \subset \mathrm{M}$ and $\mathrm{N}^{\mathrm{O}} \subset \mathrm{N}$, whose complements have codimension $\geq 2$, such that $\varphi$ is defined everywhere on $\mathrm{M}^{\mathrm{O}}, \varphi\left(\mathrm{M}^{\mathrm{O}}\right)=\mathrm{N}^{\mathrm{O}}$ and the induced morphism $\varphi^{\mathrm{O}}: \mathrm{M}^{\mathrm{O}} \rightarrow \mathrm{N}^{\mathrm{O}}$ is finite. Then $\varphi$ is everywhere defined and finite.

Proof. Replacing $\mathrm{N}$ by its normalization we may assume that $\mathrm{N}$ is normal; then the restriction maps $\mathrm{H}^{0}\left(\mathrm{~N}, \mathcal{O}_{\mathrm{N}}(n)\right) \rightarrow \mathrm{H}^{0}\left(\mathrm{~N}^{\mathrm{o}}, \mathcal{O}_{\mathrm{N}}(n)\right)$ and $\mathrm{H}^{0}\left(\mathrm{M}, \mathrm{L}^{n}\right) \rightarrow \mathrm{H}^{0}\left(\mathrm{M}^{\mathrm{o}}, \mathrm{L}^{n}\right)$ are bijective. Let $\mathrm{CM}=\operatorname{Spec} \underset{n \geq 0}{\oplus} \mathrm{H}^{0}\left(\mathrm{M}, \mathrm{L}^{n}\right)$ and $\mathrm{CN}=\operatorname{Spec} \underset{n>0}{\oplus} \mathrm{H}^{0}\left(\mathrm{~N}, \mathcal{O}_{\mathrm{N}}(n)\right)$ be the cones over $\mathrm{M}$ and $\mathrm{N}$ respectively associated to the line bundles $\mathrm{L}$ and $\mathcal{O}_{\mathrm{N}}(1)$. The homomorphism $\left(\varphi^{\mathrm{O}}\right)^{*}$ induces a finite morphism $\mathrm{C} \varphi: \mathrm{CM} \rightarrow \mathrm{CN}$, which is $\mathbf{C}^{*}$-equivariant. The inverse image of the vertex of $\mathrm{CN}$ under $\mathrm{C} \varphi$ is finite and stable under $\mathbf{C}^{*}$, hence reduced to the vertex of $\mathrm{CM}$. Therefore $\mathrm{C} \varphi$ induces a finite morphism $\mathrm{M} \rightarrow \mathrm{N}$ which extends $\varphi^{\mathrm{o}}$.

(3.4) Let us now assume that $\mathfrak{g}$ is reductive (this is our hypothesis (H2)). By (3.1) this actually implies that $\mathfrak{g}$ is semi-simple. We will always identify $\mathfrak{g}^{*}$ with $\mathfrak{g}$ using the Killing form. We also make a third hypothesis:

(H3) $\operatorname{Pic}(\mathrm{M})=\mathbf{Z}$.

This is innocuous because Theorem 0.1 is known to be true when $b_{2} \geq 2$, as a consequence of a theorem of Wisniewski (see [L-S], cor. 4.2).

Proposition 3.5. Under the hypotheses (H1) to (H3), the map $\varphi: \mathrm{M} \rightarrow \mathbf{P}(\mathfrak{g})$ is a finite morphism onto the closure of a nilpotent orbit $\mathbf{P O} . \mathrm{M}$ has only finitely many orbits; each orbit is a finite étale covering of a nilpotent orbit in $\mathbf{P}(\mathfrak{g})$.

Proof. Since G is semi-simple, the hypotheses of lemma 3.2 hold. We have already seen that the orbit $\mathcal{O}$ is $\mathbf{C}^{*}$-invariant, hence nilpotent (2.4). Therefore $\overline{\mathbf{P O}}$ is a 
finite union of nilpotent orbits in $\mathbf{P}(\mathfrak{g})$. Since such an orbit is odd-dimensional, the codimension of $\mathbf{P O}$ in $\overline{\mathbf{P O}}$ is $\geq 2$, so we can apply lemma 3.3; the Proposition follows.

Remark 3.6. Conversely, suppose given a compact manifold $M$ with an action of $\mathrm{G}$ and a finite surjective G-equivariant morphism $\varphi: \mathrm{M} \rightarrow \overline{\mathbf{P O}}$ onto the closure of a nilpotent orbit in $\mathbf{P}(\mathfrak{g})$. Then $\mathrm{M}$ is a Fano contact manifold. Indeed, let $\mathrm{M}^{\mathrm{O}}=\varphi^{-1}(\mathbf{P} \mathcal{O})$, and $\mathrm{L}=\varphi^{*} \mathcal{O}(1)$. The contact structure of $\mathbf{P} \mathcal{O}$ pulls back to a contact structure $\theta^{\mathrm{o}} \in \mathrm{H}^{0}\left(\mathrm{M}^{\mathrm{o}}, \Omega_{\mathrm{M}^{\mathrm{o}}}^{1} \otimes \mathrm{L}\right)$, which extends to a contact structure $\theta \in \mathrm{H}^{0}\left(\mathrm{M}, \Omega_{\mathrm{M}}^{1} \otimes \mathrm{L}\right)$ because $\mathrm{M}-\mathrm{M}^{\mathrm{O}}$ has codimension $\geq 2$. Since $\mathrm{L}$ is ample, $\mathrm{M}$ is a Fano contact manifold.

We have thus reduced our problem to a question about nilpotent orbits of semi-simple Lie algebras, which we will study in the next sections.

\section{Nilpotent orbits}

(4.1) At this point we need to recall Dynkin's classification of nilpotent orbits in a semi-simple Lie algebra $\mathfrak{g}$ (a general reference for the material in this section is $[\mathrm{C}-\mathrm{M}])$. We fix a nilpotent element $N_{0}$ of $\mathfrak{g}$, and denote by $\mathcal{O}$ its orbit in $\mathfrak{g}$ (under the adjoint action).

By the Jacobson-Morozov theorem, there exist elements $H$ and $N_{1}$ in $\mathfrak{g}$ satisfying

$$
\left[H, N_{0}\right]=2 N_{0} \quad\left[H, N_{1}\right]=-2 N_{1} \quad\left[N_{1}, N_{0}\right]=H,
$$

so that the subspace of $\mathfrak{g}$ spanned by $N_{0}, N_{1}, H$ is a Lie subalgebra isomorphic to $\mathfrak{s l}_{2}$. As a $\mathfrak{s l}_{2}$-module, $\mathfrak{g}$ is then isomorphic to a direct sum of simple modules $\mathrm{S}^{k} \mathrm{~V}$, where $\mathrm{V}$ is the standard 2-dimensional representation. It follows easily that:

(4.1.a) there is a direct sum decomposition $\mathfrak{g}=\underset{i \in \mathbf{Z}}{\oplus} \mathfrak{g}(i)$, where $\mathfrak{g}(i)$ is the subspace of elements $X \in \mathfrak{g}$ with $[H, X]=i X$.

(4.1.b) Put $\mathfrak{p}=\underset{i \geq 0}{\oplus} \mathfrak{g}(i), \mathfrak{n}=\underset{i \geq 2}{\oplus} \mathfrak{g}(i)$. Then $\mathfrak{p}$ is a parabolic subalgebra of $\mathfrak{g}$; $\mathfrak{n}$ is a nilpotent ideal in $\mathfrak{p}$. The map $\operatorname{ad}\left(N_{0}\right): \mathfrak{p} \rightarrow \mathfrak{n}$ is surjective.

(4.1.c) Let $\mathfrak{h}$ be a Cartan subalgebra of $\mathfrak{g}$ containing $H$. There exists a basis $\mathrm{B}$ of the root system $\mathrm{R}(\mathfrak{g}, \mathfrak{h})$ such that $\alpha(H) \in\{0,1,2\}$ for each $\alpha \in \mathrm{B}$. The weighted Dynkin diagram of $N_{0}$ is obtained by labelling each node $\alpha \in \mathrm{B}$ of the Dynkin diagram of $\mathfrak{g}$ with the number $\alpha(H) \in\{0,1,2\}$. It depends only on the orbit $\mathcal{O}$ of $N_{0}$; two different nilpotent orbits give rise to different weighted diagrams.

(4.2) Let $\mathrm{P}$ be the parabolic subgroup of $\mathrm{G}$ with Lie algebra $\mathfrak{p}$. We denote by $\mathrm{G} \times{ }^{\mathrm{P}} \mathfrak{n}$ the quotient of $\mathrm{G} \times \mathfrak{n}$ by $\mathrm{P}$ acting by $p \cdot(g, N)=\left(g p^{-1}, \operatorname{Ad}(p) N\right)$; in other words, $\mathrm{G} \times{ }^{\mathrm{P}} \mathfrak{n}$ is the G-homogeneous vector bundle on $\mathrm{G} / \mathrm{P}$ associated 
to the adjoint action of $\mathrm{P}$ on $\mathfrak{n}$. For $g \in \mathrm{G}, N \in \mathfrak{n}$, we denote by $(g, N)^{\bullet}$ the image of $(g, N)$ in $\mathrm{G} \times{ }^{\mathrm{P}} \mathfrak{n}$; the tangent space to $\mathrm{G} \times{ }^{\mathrm{P}} \mathfrak{n}$ at $(g, N)^{\circ}$ is canonically isomorphic to the quotient of $\mathfrak{g} \times \mathfrak{n}$ by the subspace of elements $(,[N]$,$) with$ $\in \mathfrak{p}$.

The orbit $\mathrm{G} \cdot\left(1, N_{0}\right)^{\cdot}$ is open in $\mathrm{G} \times{ }^{\mathrm{P}} \mathfrak{n}$. Since the stabilizer in $\mathrm{G}$ of $\left(1, N_{0}\right)^{\text {. }}$ is $\mathrm{Z}_{N_{0}}$, there is a unique G-equivariant isomorphism $\mathcal{O} \stackrel{\sim}{\longrightarrow} \mathrm{G} \cdot\left(1, N_{0}\right)$ mapping $N_{0}$ onto $\left(1, N_{0}\right)$. We will identify $\mathcal{O}$ to the open orbit of $\mathrm{G} \times{ }^{\mathrm{P}} \mathfrak{n}$ through this isomorphism. The following lemma is due to D. I. Panyushev [P] (I am indebted to the referee for pointing out this reference).

Lemma 4.3. The Kostant-Kirillov symplectic 2 -form on $\mathcal{O}$ extends to a $\mathrm{G}$ invariant 2-form $\omega$ on $\mathrm{G} \times{ }^{\mathrm{P}} \mathfrak{n}$. Let $(g, N)^{*} \in \mathrm{G} \times{ }^{\mathrm{P}} \mathfrak{n}$; the kernel of $\omega_{(g, N)}$ consists of the images of the elements $(X,[N, X])$, with $X \in \mathfrak{n}^{\perp}=\underset{i \geq-1}{\oplus} \mathfrak{g}(i)$ and $[N, X] \in \mathfrak{n}$.

Proof. Consider the alternate bilinear form on $\mathfrak{g} \times \mathfrak{n}$ defined by

$$
\left((X, Q),\left(X^{\prime}, Q^{\prime}\right)\right) \mapsto\left(N \mid\left[X, X^{\prime}\right]\right)+\left(X \mid Q^{\prime}\right)-\left(X^{\prime} \mid Q\right),
$$

where $(\mid)$ stands for the Killing form. Its kernel consists of pairs $(X, Q)$ with $X \in \mathfrak{n}^{\perp}$ and $Q=[N, X]$; in particular, it contains the elements ( , [N, ]) for $\in \mathfrak{p}$, so that our form factors through $\mathrm{T}_{(g, N)}\left(\mathrm{G} \times{ }^{\mathrm{P}} \mathfrak{n}\right)$ and defines a G-invariant 2-form $\omega$ on $\mathrm{G} \times{ }^{\mathrm{P}} \mathfrak{n}$.

The isomorphism $\mathcal{O} \rightarrow \mathrm{G} \cdot\left(1, N_{0}\right)^{\bullet}$ induces on the tangent spaces the isomorphism $\mathfrak{g} / \mathfrak{z}_{N_{0}} \rightarrow \mathrm{T}_{\left(1, N_{0}\right)} \cdot\left(\mathrm{G} \times{ }^{\mathrm{P}} \mathfrak{n}\right)$ which maps the class of $X \in \mathfrak{g}$ to the class of $(X, 0)$. Through this isomorphism, $\omega_{\left(1, N_{0}\right)}$ corresponds to the alternate form $\left(X, X^{\prime}\right) \mapsto\left(N_{0} \mid\left[X, X^{\prime}\right]\right)$, that is to the Kostant-Kirillov 2 -form $\omega_{0}$ at $N_{0}$. Since $\omega$ and $\omega_{0}$ are G-invariant, the restriction of $\omega$ to $\mathcal{O}$ is equal to $\omega_{0}$.

The following lemma will be the key technical ingredient for our proof of the main theorem. We put $\mathfrak{g}^{\times}=\mathfrak{g}-\{0\}, \mathfrak{n}^{\times}=\mathfrak{n}-\{0\}$.

Lemma 4.4. Let $N \in \mathfrak{n}$. Let $\overline{\mathcal{O}}$ be the closure of $\mathcal{O}$ in $\mathfrak{g}^{\times}$. Assume that the normalization $\widetilde{\mathcal{O}}$ of $\overline{\mathcal{O}}$ is smooth above $N$. Then the centralizer $\mathfrak{z}_{N}$ is contained in $\mathfrak{n}^{\perp}$.

Proof. Consider the morphism $\mathrm{G} \times{ }^{\mathrm{P}} \mathfrak{n}^{\times} \rightarrow \mathfrak{g}^{\times}$which maps $(g, N)^{\cdot}$ to $\operatorname{Ad}(g) N$; it is proper. Its image is the closure $\overline{\mathcal{O}}$ of $\mathcal{O}$ in $\mathfrak{g}^{\times}$; since $\mathrm{G} \underset{\widetilde{\mathcal{O}}}{ }{ }^{\mathrm{P}} \mathfrak{n}$ is smooth, it factors through $\widetilde{\mathcal{O}}$. The induced morphism $\pi: \mathrm{G} \times{ }^{\mathrm{P}} \mathfrak{n}^{\times} \rightarrow \widetilde{\mathcal{O}}$ is proper and birational: it induces the identity on the open orbit $\mathcal{O} \subset \mathrm{G} \times{ }^{\mathrm{P}} \mathfrak{n}^{\times}$.

Since the complement of $\mathcal{O}$ in $\widetilde{\mathcal{O}}$ has codimension $\geq 2$, the symplectic 2 -form on $\mathcal{O}$ extends to a 2 -form $\varpi$ on the smooth part $\widetilde{\mathcal{O}}_{s m}$ of $\widetilde{\mathcal{O}}$; the pull-back of $\varpi$ to $\pi^{-1}\left(\widetilde{\mathcal{O}}_{s m}\right) \subset \mathrm{G} \times{ }^{\mathrm{P}} \mathfrak{n}$ coincides with the restriction of $\omega$. It follows that every 
tangent vector at the point $x=(1, N)^{\cdot}$ of $\mathrm{G} \times{ }^{\mathrm{P}} \mathfrak{n}^{\times}$killed by $\mathrm{T}_{x}(\pi)$ belongs to the kernel of $\omega_{x}$. Since the orbit of $x$ under $\mathrm{Z}_{N}^{\mathrm{o}}$ maps to a point in $\widetilde{\mathcal{O}}$, the vectors $(, 0)$ with $\in \mathfrak{z}_{N}$ must belong to the kernel of $\omega_{x}$; in view of Lemma 4.3 , this means that $\mathfrak{z}_{N}$ is contained in $\mathfrak{n}^{\perp}$.

\section{The birational case}

In this section we will prove Theorem 0.1 in the simpler case when the map $\varphi_{\mathrm{L}}$ is assumed to be birational. We start with a technical lemma about Lie algebras; we keep the notation of (4.1).

Lemma 5.1. Assume that $N_{0}$ is not contained in a proper ideal of $\mathfrak{g}$, and that for every nonzero elements $N \in \mathfrak{g}(2)$ and $Q \in \mathfrak{g}(-2)$ the bracket $[N, Q]$ is nonzero. Then $\mathfrak{g}$ is simple, and either $\mathcal{O}$ is the minimal orbit, or $\mathfrak{g}$ is of type $\mathrm{G}_{2}$ and $\mathcal{O}$ is the orbit of a short root vector.

Proof. Assume first that $\mathfrak{g}$ is a product of two nonzero semi-simple Lie algebras $\mathfrak{g}^{\prime}$ and $\mathfrak{g}^{\prime \prime}$. Write $N_{0}=\left(N_{0}^{\prime}, N_{0}^{\prime \prime}\right), H=\left(H^{\prime}, H^{\prime \prime}\right), N_{1}=\left(N_{1}^{\prime}, N_{1}^{\prime \prime}\right)$; the hypothesis on $N_{0}$ ensures that $N_{0}^{\prime}$ and $N_{0}^{\prime \prime}$ (and therefore also $H^{\prime}, H^{\prime \prime}, N_{1}^{\prime}, N_{1}^{\prime \prime}$ ) are nonzero. We have $N_{1}^{\prime} \in \mathfrak{g}(-2), N_{0}^{\prime \prime} \in \mathfrak{g}(2)$ and $\left[N_{1}^{\prime}, N_{0}^{\prime \prime}\right]=0$, contrary to the hypothesis. Thus $\mathfrak{g}$ is simple.

For any nonzero $N \in \mathfrak{g}(2)$, we have $\mathfrak{z}_{N} \cap \mathfrak{g}(-2)=(0)$; by [C-M], 3.4.17, this implies that $N$ is conjugate to $N_{0}$. There exists a root $\alpha$ with $\alpha(H)=2$ (the corresponding root vectors span $\mathfrak{g}(2))$; therefore $N_{0}$ is conjugate to $X_{\alpha}$.

Assume that $\mathfrak{g}$ is of type $\mathrm{B}_{l}, \mathrm{C}_{l}$ or $\mathrm{F}_{4}$, and that $\alpha$ is a short root. According to $[\mathrm{C}-\mathrm{M}]$ the weighted Dynkin diagram of $X_{\alpha}$ is one of the following:

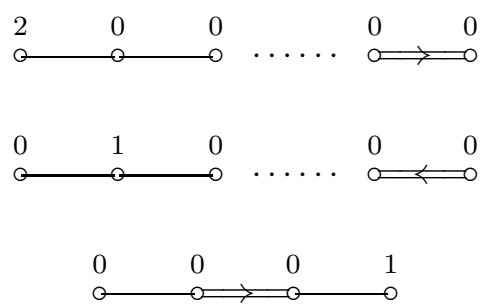

In each case the highest root $\theta$ satisfies $\theta(H)=2$, hence $X_{\theta}$ should be conjugate to $X_{\alpha}$ - a contradiction. Therefore either $\alpha$ is a long root, or $\mathfrak{g}$ is of type $\mathrm{G}_{2}$.

Proposition 5.2. Let $\mathcal{O}$ be a nilpotent orbit in $\mathfrak{g}$ and $\overline{\mathcal{O}}$ its closure in $\mathfrak{g}^{\times}$. Assume that $\mathcal{O}$ is not contained in a proper ideal of $\mathfrak{g}$, and that the normalization 
of $\overline{\mathcal{O}}$ is smooth. Then $\mathfrak{g}$ is simple, and either $\mathcal{O}$ is the minimal nilpotent orbit, or $\mathfrak{g}$ is of type $\mathrm{G}_{2}$ and $\mathcal{O}$ is the orbit of a short root vector.

In the first case $\overline{\mathcal{O}}$ is equal to $\mathcal{O}$, hence smooth. In the second case $\overline{\mathcal{O}}$ is not normal, and its normalization is isomorphic to the minimal nilpotent orbit in $\mathfrak{s o}(7)[\mathrm{L}-\mathrm{Sm}]$.

Proof. By Lemma 4.4, we have $\mathfrak{z}_{N} \subset \mathfrak{n}^{\perp}$ for each nonzero element $N$ of $\mathfrak{n}$. Taking $N$ in $\mathfrak{g}(2)$, we see that the hypotheses of Lemma 5.1 are satisfied, hence the result.

Corollary 5.3. Let $\mathrm{M}$ be a Fano contact manifold, such that

(i) the rational map $\varphi: \mathrm{M} \rightarrow \mathbf{P}(\mathfrak{g})$ is generically injective;

(ii) the group $\mathrm{G}$ of contact automorphisms of $\mathrm{M}$ is reductive.

Then $\varphi$ induces an isomorphism of $\mathrm{M}$ onto the minimal nilpotent orbit in $\mathbf{P}(\mathfrak{g})$.

Proof. Consider the commutative diagram (1.5)

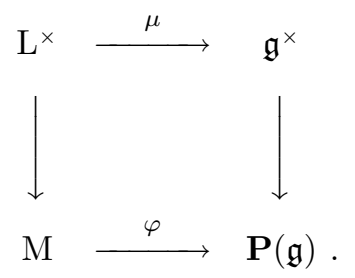

By Prop. $3.5 \varphi$ is a finite birational morphism onto the closure of a nilpotent orbit $\mathbf{P O}$ in $\mathbf{P}(\mathfrak{g})$; since the diagram is cartesian, $\mu$ is finite and birational onto $\overline{\mathcal{O}}$, hence realizes $\mathrm{L}^{\times}$as the normalization of $\overline{\mathcal{O}}$. Since the image $\overline{\mathbf{P O}}$ of $\varphi$ spans $\mathbf{P}(\mathfrak{g}), \mathcal{O}$ cannot be contained in any proper subspace of $\mathfrak{g}$. By Prop. 5.2, this implies either that $\mathcal{O}$ is a minimal orbit, or that $\mathfrak{g}$ is of type $\mathrm{G}_{2}$ and $\mathcal{O}$ is the orbit of a short root vector; in that case $\mathrm{M}$ is isomorphic to $\mathbf{P} \mathcal{O}^{\prime}$, where $\mathcal{O}^{\prime}$ is the minimal orbit in $\mathfrak{s o}(7)$, and this isomorphism preserves the contact structures (remark 2.8). But then $\mathfrak{g}$ contains $\mathfrak{s o}(7)$, a contradiction.

\section{The general case}

(6.1) As explained in Remark 3.6, we want to classify finite G-equivariant surjective morphisms $\varphi: \mathrm{M} \rightarrow \overline{\mathbf{P O}}$, where $\mathrm{M}$ is smooth and $\mathcal{O} \subset \mathfrak{g}$ is a nilpotent orbit; such a morphism will be called for short a G-covering of $\overline{\mathbf{P O}}$. Examples of 
G-coverings appear in the classification of "shared orbit pairs" [B-K], associated to certain pairs $\mathfrak{g} \subset \mathfrak{g}^{\prime}$ of simple Lie algebras: the manifold $\mathrm{M}$ is the minimal orbit $\mathbf{P} \mathcal{O}_{\text {min }}^{\prime}$ for $\mathfrak{g}^{\prime}$, while the orbit $\mathcal{O} \subset \mathfrak{g}$ is given in the list below. Brylinski and Kostant find the following cases:

\begin{tabular}{|c|c|c|c|}
\hline $\mathfrak{g}$ & $\mathfrak{g}^{\prime}$ & $\mathcal{O}$ & $\operatorname{deg} \varphi$ \\
\hline $\mathrm{A}_{2}$ & $\mathrm{G}_{2}$ & $\mathcal{O}_{(3)}$ & 3 \\
\hline $\mathrm{B}_{l}$ & $\mathrm{D}_{l+1}$ & $\mathcal{O}_{(3,1, \ldots, 1)}$ & 2 \\
\hline $\mathrm{B}_{4}$ & $\mathrm{~F}_{4}$ & $\mathcal{O}_{(2,2,2,2,1)}$ & 2 \\
\hline $\mathrm{C}_{l}$ & $\mathrm{~A}_{2 l-1}$ & $\mathcal{O}_{(2,2,1, \ldots, 1)}$ & 2 \\
\hline $\mathrm{D}_{l}$ & $\mathrm{~B}_{l}$ & $\mathcal{O}_{(3,1, \ldots, 1)}$ & 2 \\
\hline $\mathrm{D}_{4}$ & $\mathrm{~F}_{4}$ & $\mathcal{O}_{(3,2,2,1)}$ & 4 \\
\hline $\mathrm{F}_{4}$ & $\mathrm{E}_{6}$ & $\mathcal{O}_{\text {short }}$ & 2 \\
\hline $\mathrm{G}_{2}$ & $\mathrm{~B}_{3}$ & $\mathcal{O}_{\text {short }}$ & 1 \\
\hline $\mathrm{G}_{2}$ & $\mathrm{D}_{4}$ & $\mathcal{O}_{\text {sub }}$ & 6 \\
\hline
\end{tabular}

The notation for the orbit $\mathcal{O}$ requires some explanation: in the classical cases, $\mathfrak{g}$ is viewed as an algebra of matrices via the standard representation; then $\mathcal{O}_{\left(d_{1}, \ldots, d_{k}\right)}$ denote the conjugacy class of a matrix in $\mathfrak{g}$ with Jordan type $\left(d_{1}, \ldots, d_{k}\right)$. As in $(2.5), \mathcal{O}_{\text {short }}$ is the orbit of a short root vector. Finally $\mathcal{O}_{\text {sub }}$ is the so-called subregular orbit, that is the unique codimension 2 orbit in the nilpotent cone.

Proposition 6.3. Let $\mathrm{G}$ be a simple complex Lie group acting on a manifold $\mathrm{M}, \mathfrak{g}$ the Lie algebra of $\mathrm{G}, \mathcal{O} \subset \mathfrak{g}$ a nilpotent orbit, $\varphi: \mathrm{M} \rightarrow \overline{\mathbf{P O}}$ a finite $\mathrm{G}$ equivariant surjective morphism. Then either $\mathcal{O}=\mathcal{O}_{\text {min }}$ and $\varphi$ is an isomorphism, or $\varphi$ is (up to isomorphism) one of the $\mathrm{G}$-coverings appearing in the list $(6.2)$.

Proof. (6.4) Let $\mathrm{M}^{\mathrm{O}}$ be the open G-orbit in $\mathrm{M}$; let $m$ be a point of $\mathrm{M}^{\mathrm{O}}, \mathrm{H}^{\mathrm{O}}$ its stabilizer in $\mathrm{G}$ and $\mathrm{H}$ the stabilizer of $\varphi(m)$. Since $\mathrm{M}$ is Fano, $\mathrm{M}$ and therefore $\mathrm{M}^{\mathrm{O}}$ are simply connected; this implies that $\mathrm{H}^{\mathrm{O}}$ is the neutral component of $\mathrm{H}$. So the covering $\mathrm{M}^{\mathrm{O}} \rightarrow \mathbf{P O}$ is a Galois covering, with Galois group $\Gamma:=\mathrm{H} / \mathrm{H}^{\mathrm{O}}$. Since $\mathrm{M}=$ Proj $\underset{n \geq 0}{\oplus} \mathrm{H}^{0}\left(\mathrm{M}^{\mathrm{O}}, \mathrm{L}^{n}\right)$, the action of $\Gamma$ on $\mathrm{M}^{\mathrm{o}}$ extends to an action on $\mathrm{M}$, which commutes with the G-action.

Observe that the G-covering $\mathrm{M} \rightarrow \overline{\mathbf{P O}}$ is uniquely determined by $\mathcal{O}$ : the open G-orbit $\mathrm{M}^{\mathrm{O}} \subset \mathrm{M}$ is the simply-connected covering of $\mathbf{P O}$, and $\mathrm{M}$ is the integral closure of $\overline{\mathbf{P O}}$ in $\mathrm{M}^{\mathrm{O}}$. Thus our task is to prove that only the orbits 
listed in (6.2) can occur.

(6.5) We will prove this by induction on the dimension of $\mathcal{O}$, the case $\mathcal{O}=\mathcal{O}_{\text {min }}$ being clear in view of (6.4). By Prop. 5.2 we can assume $\operatorname{deg}(\varphi)>1$. Let $\gamma \in \Gamma$, and let $\mathrm{F}$ be a component of the fixed locus of $\gamma$. Then $\mathrm{F}$ is a closed submanifold of $\mathrm{M}$, stable under $\mathrm{G}$; the map $\varphi$ induces a $\mathrm{G}$-covering $\mathrm{F} \rightarrow \overline{\mathbf{P O}}_{\mathrm{F}}$ for some orbit $\mathcal{O}_{\mathrm{F}} \subset \overline{\mathcal{O}}$. By the induction hypothesis, $\mathrm{F}$ is isomorphic to the minimal orbit $\mathbf{P} \mathcal{O}_{\text {min }}^{\prime}$ for some simple Lie algebra $\mathfrak{g}^{\prime}$ containing $\mathfrak{g}$; either $\mathfrak{g}^{\prime}=\mathfrak{g}$, or the pair $\left(\mathfrak{g}, \mathfrak{g}^{\prime}\right)$ is one of the pairs appearing in the list $(6.2)$.

Let us say for short that an orbit $\mathcal{O}^{\prime} \subset \overline{\mathcal{O}}$ is ramified if $\varphi^{-1}\left(\mathbf{P} \mathcal{O}^{\prime}\right)$ is contained in the fixed locus of some nontrivial element of $\Gamma$. Let $\mathcal{O}^{\prime} \subset \overline{\mathcal{O}}$ an orbit which is not ramified; since $\varphi$ induces an isomorphism of $\mathrm{M} / \Gamma$ onto the normalization $\widetilde{\mathbf{P O}}$ of $\overline{\mathbf{P O}}$, we have:

(6.5.a) $\widetilde{\mathbf{P O}}$ is smooth along $\mathbf{P} \mathcal{O}^{\prime}$; in particular, the centralizer of any element of $\mathcal{O}^{\prime} \cap \mathfrak{n}$ is contained in $\mathfrak{n}^{\perp}$ (lemma 4.4).

(6.5.b) Any nonzero element $N \in \mathcal{O}^{\prime} \cap \mathfrak{g}(2)$ satisfies $\mathfrak{z}_{N} \cap \mathfrak{g}(-2)=(0)$, hence is conjugate to $N_{0}$ by $[\mathrm{C}-\mathrm{M}], 3.4 .17$; therefore if $\mathcal{O}^{\prime} \cap \mathfrak{g}(2) \neq(0)$, then $\mathcal{O}^{\prime}=\mathcal{O}$.

(6.5.c) Assume that $\overline{\mathcal{O}}$ is normal along $\mathcal{O}^{\prime}$. Then $\varphi$ is étale above $\mathbf{P} \mathcal{O}^{\prime}$, so that $\mathrm{T}_{m}(\varphi)$ is injective at each point $m$ of $\varphi^{-1}\left(\mathbf{P} \mathcal{O}^{\prime}\right)$. But this implies that $m$ belongs to the open orbit $\mathrm{M}^{\mathrm{O}}$ (Prop. 1.7), hence $\mathcal{O}^{\prime}=\mathcal{O}$ again.

(6.5.d) Assume that the Galois group $\Gamma$ is cyclic of prime order, and that $\overline{\mathcal{O}}$ is normal. Let $\mathrm{M}^{\Gamma}$ be the fixed locus of $\Gamma$ in $\mathrm{M}$. Then $\varphi$ induces an isomorphism of $\mathrm{M}^{\Gamma}$ onto its image; in particular, $\varphi\left(\mathrm{M}^{\Gamma}\right)$ is smooth. By Prop. 5.2, this implies that the only ramified orbit is $\mathcal{O}_{\text {min }}$, so by (6.5.c) we have $\overline{\mathcal{O}}=\mathcal{O} \cup \mathcal{O}_{\text {min }}$.

(6.6) Now we examine which orbits $\mathcal{O} \subset \mathfrak{g}$ may occur. We order the nilpotent orbits by the relation " $\mathcal{O}^{\prime} \leq \mathcal{O}$ iff $\mathcal{O}^{\prime} \subset \overline{\mathcal{O}}$ ". Given the Lie algebra $\mathfrak{g}$, the possible ramified orbits are those contained in the closure of the orbit $\mathcal{O}$ in (6.2). Using the above arguments we will show that only one more orbit is allowed: its boundary must contain only ramified orbits. This gives us for each Lie algebra $\mathfrak{g}$ a small list of orbits, among which we may eliminate those which are simply connected; we will show that the remaining ones are those which appear in the list (6.2).

Type $\mathrm{A}_{l}(l \geq 4)$

All orbit closures in case $\mathrm{A}_{l}$ are normal [K-P1], so by (6.5.c) there is only one orbit which is not ramified. There is no shared orbit pair, so the only ramified orbit is the minimal one. The next orbit in the partial ordering is $\mathcal{O}_{(2,2,1, \ldots)}$, which is simply-connected [C-M, p. 92].

Type $\mathrm{A}_{3}$

The possible ramified orbits are $\mathcal{O}_{\text {min }}$ and $\mathcal{O}_{(2,2)}$; the next orbit in the partial ordering is $\mathcal{O}_{(3,1)}$. The orbit $\mathcal{O}_{(2,2)}$ gives rise to case $\left(\mathrm{D}_{3}, \mathrm{~B}_{3}\right)$ of $(6.2) ; \mathcal{O}_{(3,1)}$ is simply-connected.

Type $\mathrm{A}_{2}$

There are only two orbits, $\mathcal{O}_{\text {min }}$ and the principal orbit $\mathcal{O}_{(3)}$, which gives rise to case $\left(\mathrm{A}_{2}, \mathrm{G}_{2}\right)$ of $(6.2)$. 
For the types $\mathrm{B}_{l}, \mathrm{C}_{l}$ or $\mathrm{D}_{l}$, most orbit closures are normal, with the following exceptions [K-P2]:

a) There may exist an orbit $\mathcal{O}$ whose closure is non-normal along a codimension 2 orbit $\mathcal{O}^{\prime}$, but whose normalization is singular along $\mathcal{O}^{\prime}$. In this case by (6.5.a) $\mathcal{O}^{\prime}$ is ramified;

b) When $\mathfrak{g}$ is of type $\mathrm{D}_{l}$, there are orbits (corresponding to the so-called "very even" classes) whose closure is not known to be normal. However these orbit closures have a boundary component of codimension 2 along which they are normal, so that (6.5.c) still applies.

Type $\mathrm{B}_{l}$ and $\mathrm{D}_{l}, l \geq 5$

The Lie algebra $\mathfrak{g}$ is $\mathfrak{s o}(n) \quad(n \geq 10)$. The possible ramified orbits are $\mathcal{O}_{\text {min }}$ and $\mathcal{O}_{(3,1, \ldots)}$; the only possible next orbit is $\mathcal{O}_{(2,2,2,2,1, \ldots)}\left(\mathcal{O}_{(3,2,2,1, \ldots)}\right.$ is excluded because its closure contains $\mathcal{O}_{(2,2,2,2,1, \ldots)}$ which is not ramified). The orbit $\mathcal{O}_{(3,1, \ldots)}$ gives rise to cases $\left(\mathrm{B}_{l}, \mathrm{D}_{l+1}\right)$ and $\left(\mathrm{D}_{l}, \mathrm{~B}_{l}\right) ; \mathcal{O}_{(2,2,2,2,1, \ldots)}$ is simplyconnected ([C-M], p. 92).

Type $\mathrm{B}_{4}$

The configuration of orbits is the same as above, but here the orbit $\mathcal{O}_{(2,2,2,2,1)}$ can be ramified. Therefore the next orbit $\mathcal{O}_{(3,2,2,1,1)}$ might occur. However its fundamental group is $\mathbf{Z} / 2$, and its closure is normal [K-P2], so we deduce from (6.5.d) that this orbit does not occur.

The orbit $\mathcal{O}_{(2,2,2,2,1)}$ is no longer simply-connected; it gives rise to case $\left(\mathrm{B}_{4}, \mathrm{~F}_{4}\right)$ in $(6.2)$.

Type $\mathrm{B}_{3}$

Again the orbit $\mathcal{O}_{(3,2,2)}$ can occur a priori; the same argument as for $\mathrm{B}_{4}$ applies.

Type $\mathrm{D}_{4}$

The possible ramified orbits are $\mathcal{O}_{\text {min }}$, the three orbits next to $\mathcal{O}_{\text {min }}$ in the partial ordering (namely $\mathcal{O}_{(3,1, \ldots)}$ and the two orbits $\left.\mathcal{O}_{(2,2,2,2)}\right)$, and $\mathcal{O}_{(3,2,2,1)}$; the next orbit is $\mathcal{O}_{(3,3,1,1)}$.

The three orbits next to $\mathcal{O}_{\min }$ have the same weighted Dynkin diagram up to automorphisms, and are therefore isomorphic; they give the case $\left(\mathrm{D}_{4}, \mathrm{~B}_{4}\right)$. The orbit $\mathcal{O}_{(3,2,2,1)}$ gives the case $\left(\mathrm{D}_{4}, \mathrm{~F}_{4}\right)$. Finally $\mathcal{O}_{(3,3,1,1)}$ has fundamental group $\mathbf{Z} / 2$ and normal closure [K-P2], so is excluded by (6.5.d).

Type $\mathrm{C}_{l} \quad(l \geq 2)$

The possible ramified orbits are $\mathcal{O}_{\min }$ and $\mathcal{O}_{(2,2,1, \ldots)}$; the next orbit is $\mathcal{O}_{(2,2,2,1, \ldots)}$ if $l \geq 3$, and $\mathcal{O}_{(4)}$ if $l=2$. This orbit has fundamental group $\mathbf{Z} / 2$ and is normal $[\mathrm{K}-\mathrm{P} 2]$, so it is excluded again by $(6.5 . d)$. The orbit $\mathcal{O}_{(2,2,1, \ldots)}$ gives the case $\left(\mathrm{C}_{l}, \mathrm{~A}_{2 l-1}\right)$.

Type $\mathrm{E}_{l}$

The only possible ramified orbit is the minimal one. If $\mathcal{O}$ is not reduced to $\mathcal{O}_{\min }$ it contains the next orbit $\mathcal{O}_{1}$ in the partial ordering, which is the orbit of 
$X_{\lambda}+X_{\mu}$, where $\lambda$ and $\mu$ are two orthogonal roots. By (6.5.a) the centralizer of an element of $\mathcal{O}_{1} \cap \mathfrak{n}$ is contained in $\mathfrak{n}^{\perp}$.

Let $\sigma$ be the sum of the simple roots, and $\alpha, \beta, \gamma$ the simple roots corresponding to the three ends of the Dynkin graph. Then $\sigma, \sigma-\alpha, \sigma-\beta, \sigma-\gamma$ are roots $\left([\mathrm{B}], \S 1, \mathrm{n}^{\mathrm{o}} 6\right.$, cor. 3 of prop. 19); since $(\sigma \mid \sigma)=2$ and $(\sigma \mid \alpha)=(\sigma \mid \beta)=1$, $\sigma-\alpha$ and $\sigma-\beta$ are orthogonal. The element $N=X_{\sigma-\alpha}+X_{\sigma-\beta}$ satisfies $\left[N, X_{\gamma-\sigma}\right]=0$. Let $s=\sigma(H)$ and $m=\max \{\alpha(H), \beta(H), \gamma(H)\}$. If $s-m \geq 2$ we have $N \in \mathfrak{n}$ and $X_{\gamma-\sigma} \notin \mathfrak{n}^{\perp}$, a contradiction.

Suppose $s=2$ and $\alpha(H)=\beta(H)=0$. Then $N$ belongs to $\mathfrak{g}(2)$, which by (6.5.b) implies $\mathcal{O}=\mathcal{O}_{1}$; this is excluded because $\mathcal{O}_{1}$ is simply-connected ([C-M], pp. 129, 130, 132).

Looking at the list of possible weighted Dynkin diagrams in loc. cit. and eliminating the simply-connected orbits, the above constraints leave us with only one possible case, the weighted Dynkin diagram

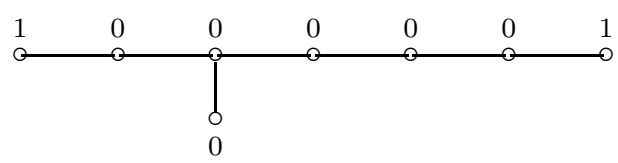

for $\mathrm{E}_{8}$. In that case one finds easily two orthogonal roots $\lambda$ and $\mu$ with $\lambda(H)=\mu(H)=2$, for instance (with the notation of [B], planche VII) $\lambda=\frac{1}{2} \sum_{i} \varepsilon_{i}$ and $\mu=\varepsilon_{8}-\varepsilon_{7}$; we conclude again by (6.5.b) that $\mathcal{O}=\mathcal{O}_{1}$.

Type $\mathrm{F}_{4}$

The orbits which can be ramified are $\mathcal{O}_{\text {min }}$ and $\mathcal{O}_{\text {short }}$. If $\mathcal{O}$ is bigger than $\mathcal{O}_{\text {short }}$, it contains the orbit $\mathcal{O}_{1}$ next to $\mathcal{O}_{\text {short }}$; this is the orbit of $X_{\alpha}+X_{\beta}$, where $\alpha$ and $\beta$ are two orthogonal roots of distinct lengths. Let

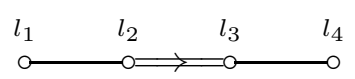

be the weighted Dynkin diagram of $\mathcal{O}$. Assume first $l_{1}+l_{2}+l_{3} \geq 2$. Using the notation of [B], planche VIII, let

$$
\begin{gathered}
\alpha=\varepsilon_{2}=\alpha_{1}+\alpha_{2}+\alpha_{3}, \quad \beta=\varepsilon_{1}-\varepsilon_{4}=\alpha_{1}+2 \alpha_{2}+2 \alpha_{3}+2 \alpha_{4}, \\
\gamma=\varepsilon_{1}+\varepsilon_{4}=\alpha_{1}+2 \alpha_{2}+4 \alpha_{3}+2 \alpha_{4} .
\end{gathered}
$$

We have $\left[X_{\alpha}+X_{\beta}, X_{-\gamma}\right]=0, X_{\alpha}+X_{\beta} \in \mathfrak{n}$ and $X_{-\gamma} \notin \mathfrak{n}^{\perp}$, contradicting (6.5.a).

A glance at the tables $([\mathrm{C}-\mathrm{M}]$, p. 128$)$ shows that the nilpotent orbits with $l_{1}+l_{2}+l_{3} \leq 1$ are simply-connected, with the exception of $\mathcal{O}_{\text {short }}$; the latter gives the case $\left(\mathrm{F}_{4}, \mathrm{E}_{6}\right)$. 
Type $\mathrm{G}_{2}$

The only orbit which is not simply-connected is the subregular orbit ([C-M], p. 128), which gives rise to case $\left(\mathrm{G}_{2}, \mathrm{D}_{4}\right)$.

Example 6.7. Let us give an example of a G-covering when $\mathfrak{g}$ is not simple. Let $\mathbf{n}=\left(n_{1}, \ldots, n_{k}\right)$ be a sequence of positive integers; for each $i$, let $\mathfrak{g}_{i}$ be the Lie algebra $\mathfrak{s p}\left(2 n_{i}\right)$, and $\mathrm{V}_{i}\left(\cong \mathbf{C}^{2 n_{i}}\right)$ its standard representation. Then $\mathfrak{g}_{i}$ can be identified with $\mathrm{S}^{2} \mathrm{~V}_{i}$; the minimal nilpotent orbit $\mathcal{O}_{i} \subset \mathfrak{g}_{i}$ is then identified with the cone of rank one tensors, so that we have a 2-to-1 map $\mu_{i}: \mathrm{V}_{i} \rightarrow \overline{\mathcal{O}}_{i}=\mathcal{O}_{i} \cup\{0\}$ mapping a vector $v$ to $v^{2}$. We put $\mathfrak{g}=\prod_{i} \mathfrak{g}_{i}, \mathcal{O}=\prod_{i} \mathcal{O}_{i}, \quad \mathrm{M}=\mathbf{P}(\mathrm{V})$ with $\mathrm{V}=\oplus_{i} \mathrm{~V}_{i}$. The maps $\mu_{i}$ define a G-covering $\varphi_{\mathbf{n}}: \mathbf{P}(\mathrm{V}) \rightarrow \overline{\mathbf{P O}}$, of degree $2^{k-1}$. Note that $M$ is a minimal orbit in $\mathbf{P}\left(\mathfrak{g}^{\prime}\right)$, with $\mathfrak{g}^{\prime}=\mathfrak{s p}(\mathrm{V})$.

Proposition 6.8. Assume that $\mathfrak{g}$ is a product of simple Lie algebras $\mathfrak{g}_{1}, \ldots, \mathfrak{g}_{k}$ $(k>1)$. Let $\varphi: \mathrm{M} \rightarrow \overline{\mathbf{P O}}$ be a $\mathrm{G}$-covering. Then there exists a sequence $\mathbf{n}=$ $\left(n_{1}, \ldots, n_{k}\right)$ of positive integers such that $\varphi$ is isomorphic to the G-covering $\varphi_{\mathbf{n}}$ of example 6.7. In particular, $\mathfrak{g}_{i}$ is isomorphic to $\mathfrak{s p}\left(2 n_{i}\right)$ for each $i$, the orbit $\mathcal{O}$ is the product of the minimal orbits $\mathcal{O}_{i} \subset \mathfrak{g}_{i}$, and $\mathrm{M}$ is isomorphic to $\mathbf{P}^{2 n-1}$ with $n=\sum n_{i}$.

Proof. The orbit $\mathcal{O}$ is a product of nontrivial orbits $\mathcal{O}_{i} \subset \mathfrak{g}_{i}$. Let $\mathcal{O}_{i}^{s c}$ be the simply-connected covering of $\mathcal{O}_{i}$, and $\overline{\mathcal{O}}_{i}^{s c}$ the integral closure of $\overline{\mathcal{O}}_{i}$ in $\mathcal{O}_{i}^{s c}$ (contrary to an earlier notation, we denote by $\overline{\mathcal{O}}_{i}$ the closure of $\mathcal{O}_{i}$ in $\mathfrak{g}$ ). The action of $\mathrm{G} \times \mathbf{C}^{*}$ on $\mathcal{O}_{i}$ extends to an action on $\mathcal{O}_{i}^{s c}$ and $\overline{\mathcal{O}_{i}^{s c}}$. There is only one point $o_{i}$ of $\overline{\mathcal{O}_{i}^{s c}}$ above $0 \in \mathfrak{g}$; the open subset $\overline{\mathcal{O}_{i}^{s c}}-\left\{o_{i}\right\}$ is a principal $\mathrm{C}^{*}$-bundle over a variety $\mathrm{M}_{i}$ which admits a finite $\mathrm{G}$-equivariant morphism onto $\overline{\mathbf{P O}}_{i}$.

Let $\mathrm{M}^{\prime}=\left(\prod_{i} \overline{\mathcal{O}_{i}^{s c}}\right)^{\times} / \mathbf{C}^{*}$, where the superscript $\times$ means that we take out the point $\left(o_{1}, \ldots, o_{k}\right)$. This is a normal variety, with a finite morphism onto $\overline{\mathbf{P O}}$; the open subset $\left(\prod \mathcal{O}_{i}^{s c}\right) / \mathbf{C}^{*}$ is simply-connected and its complement has codimension $\geq 2$. This implies that $M^{\prime}$ is isomorphic to $M$.

Since $\mathrm{M}$ is smooth, it follows that each $\overline{\mathcal{O}_{i}^{s c}}$ must be smooth. This implies first of all that $\mathrm{M}_{i}$ is smooth, hence by Prop. 5.2 and 6.3 isomorphic to the minimal orbit $\mathbf{P} \mathcal{O}_{i}^{\prime}$ for some simple Lie algebra $\mathfrak{g}_{i}^{\prime}$ containing $\mathfrak{g}_{i}$. Then $\mathcal{O}_{i}^{s c}$ is the simply-connected cover of $\mathcal{O}_{i}^{\prime}$, and $\overline{\mathcal{O}_{i}^{s c}}$ is its integral closure in $\overline{\mathcal{O}}_{i}$. Since $\overline{\mathcal{O}_{i}^{s c}}$ is smooth, this happens if and only if $\mathfrak{g}_{i}=\mathfrak{g}_{i}^{\prime} \cong \mathfrak{s p}\left(2 n_{i}\right)$ for some integer $n_{i} \geq 1$ ([B-K], thm. 4.6); then $\mathcal{O}_{i}=\mathcal{O}_{i}^{\prime}$ by Prop. 6.3 , so we are in the situation of example 6.7 .

The above results imply directly Theorem 0.1 , in a slightly more precise form: 
Theorem 6.9. Let $\mathrm{M}$ be a Fano contact manifold, satifying the conditions (H1) and (H2) of Theorem 0.1. Then the Lie algebra $\mathfrak{g}$ of infinitesimal contact transformations of $\mathrm{M}$ is simple, and the canonical map $\varphi: \mathrm{M} \rightarrow \mathbf{P}(\mathfrak{g})$ induces an isomorphism of $\mathrm{M}$ onto the minimal orbit $\mathbf{P} \mathcal{O}_{\min } \subset \mathbf{P}(\mathfrak{g})$.

Proof. By (3.4), we can assume that M satisfies also (H3); then $\varphi$ induces a G-covering $\mathrm{M} \rightarrow \overline{\mathbf{P O}}$ onto the closure of some nilpotent orbit in $\mathbf{P}(\mathfrak{g})$ (Prop. 3.5). By Prop. 6.3 and 6.8, $\mathrm{M}$ is isomorphic to the minimal orbit in $\mathbf{P}\left(\mathfrak{g}^{\prime}\right)$ for some simple Lie algebra $\mathfrak{g}^{\prime}$ containing $\mathfrak{g}$; moreover if $\varphi$ is not an embedding, $\mathfrak{g}^{\prime}$ contains strictly $\mathfrak{g}$, which is impossible since $\mathfrak{g}^{\prime}$ is an algebra of infinitesimal contact transformations of M (see remark 2.8). Therefore $\varphi$ is an embedding and $\mathfrak{g}^{\prime}=\mathfrak{g}$.

\section{References}

[A] V. Arnold, Mathematical methods of classical mechanics, Graduate Texts in Math. 60. Springer-Verlag, New York-Heidelberg 1978.

[B] N. Bourbaki, Groupes et algèbres de Lie, Chap. VI. Hermann, Paris 1968.

[B-K] R. Brylinski and B. Kostant, Nilpotent orbits, normality and Hamiltonian group actions. J. of the A.M.S. 7 (1994), 269-298.

[C-M] D. Collingwood and W. Mc Govern, Nilpotent orbits in semi-simple Lie algebras. Van Nostrand Reinhold Co., New York 1993.

[K-P1] H. Kraft and C. Procesi, Closures of conjugacy classes of matrices are normal, Invent. math. 53 (1979), 227-247.

[K-P2] H. Kraft and C. Procesi, On the geometry of the conjugacy classes in classical groups, Comment. Math. Helvetici 57 (1982), 539-602.

[L] C. Le Brun, Fano manifolds, contact structures, and quaternionic geometry, Int. J. of Math. 6 (1995), 419-437.

[L-S] C. Le Brun and S. Salamon, Strong rigidity of quaternion-Kähler manifolds, Invent. math. 118 (1994), 109-132.

[L-Sm] T. Levasseur and S. Smith, Primitive ideals and nilpotent orbits in type $\mathrm{G}_{2}$, J. of Algebra 114 (1988), 81-105.

[M] D. Mumford and J. Fogarty, Geometric invariant theory. $2^{\text {nd }}$ edition. Springer-Verlag, New York-Heidelberg 1982.

$[\mathrm{Mu}]$ S. Mukai, Biregular classification of Fano 3-folds and Fano manifolds of coindex 3 , Proc. Nat. Acad. Sci. USA 86 (1989), 3000-3002.

[P] D. I. Panyushev, Rationality of singularities and the Gorenstein properties of nilpotent orbits, Functional Anal. Appl. 25 (1991), 225-226.

Arnaud Beauville

DMI - École Normale Supérieure

URA $762 \mathrm{du}$ CNRS

45 rue d'Ulm

F-75230 Paris Cedex 05

e-mail: beauville@dmi.ens.fr

(Received: July 28, 1997) 